\title{
Internal dynamics of microgels: A mesoscale hydrodynamic simulation study
}

Ali Ghavami, Hideki Kobayashi, and Roland G. Winkler

Citation: J. Chem. Phys. 145, 244902 (2016); doi: 10.1063/1.4972893

View online: http://dx.doi.org/10.1063/1.4972893

View Table of Contents: http://aip.scitation.org/toc/jcp/145/24

Published by the American Institute of Physics

\section{Articles you may be interested in}

Electrophoresis of Janus particles: A molecular dynamics simulation study

J. Chem. Phys. 145, 244704244704 (2016); 10.1063/1.4972522

Accurate schemes for calculation of thermodynamic properties of liquid mixtures from molecular dynamics simulations

J. Chem. Phys. 145, 244504244504 (2016); 10.1063/1.4973001

Dynamics of single semiflexible polymers in dilute solution

J. Chem. Phys. 145, 234903234903 (2016); 10.1063/1.4971861

Thermodynamic and kinetic solid-liquid interface properties from transition path sampling

J. Chem. Phys. 145, 244703244703 (2016); 10.1063/1.4972583 


\title{
Internal dynamics of microgels: A mesoscale hydrodynamic simulation study
}

\author{
Ali Ghavami, Hideki Kobayashi, and Roland G. Winkler ${ }^{\text {) }}$ \\ Institute for Advanced Simulation, Forschungszentrum Jülich GmbH, 52425 Jülich, Germany
}

(Received 26 October 2016; accepted 11 December 2016; published online 28 December 2016)

\begin{abstract}
We analyze the dynamics of polymers in a microgel system under different swelling conditions. A microgel particle consists of coarse-grained linear polymers which are tetra-functionally crosslinked and undergoes conformational changes in response to the external stimuli. Here, a broad range of microgel sizes, extending from tightly collapsed to strongly swollen particles, is considered. In order to account for hydrodynamic interactions, the microgel is embedded in a multiparticle collision dynamics fluid while hydrophobic attraction is modelled by an attractive Lennard-Jones potential and swelling of ionic microgels is described through the Debye-Hückel potential. The polymer dynamics is analyzed in terms of the monomer mean square displacement and the intermediate scattering function $S(q, t)$. The scattering function decays in a stretched-exponential manner, with a decay rate exhibiting a crossover from a collective diffusive dynamics at low magnitudes of the wavevector $q$ to a hydrodynamicdominated dynamics at larger $q$. There is little difference between the intermediate scattering functions of microgels under good solvent conditions and strongly swollen gels, but strongly collapsed gels exhibit a faster decay at short times and hydrodynamic interactions become screened. In addition, we present results for the dynamics of the crosslinks, which exhibit an unexpected, semiflexible polymer-like dynamics. Published by AIP Publishing. [http://dx.doi.org/10.1063/1.4972893]
\end{abstract}

\section{INTRODUCTION}

Responsive microgels are a unique class of materials comprised of a crosslinked polymer network. ${ }^{1,2}$ Microgels exhibit large conformational changes in solution in response to stimuli like changes in temperature, $\mathrm{pH}$, or ionic strength of the solvent. These unique features render them ideal candidates for a broad range of applications such as drug delivery, ${ }^{3,4}$ sensing, ${ }^{5}$ fabrication of photonic crystals, ${ }^{6}$ separation and purification technologies, ${ }^{7}$ and synthesis of organic nano-particles. ${ }^{8}$ For the rational design and special-purpose applications of microgels, a fundamental understanding of the internal dynamics of the polymers inside the gel is desirable.

Despite its importance, little is known about the dynamical properties of individual microgels. Due to their porous structure, microgels allow for a free penetration and passage of small solvent molecules which implies that hydrodynamic interactions (HI) play an important role in their internal dynamics. ${ }^{9}$ Additionally, the finite size of microgels may result in processes that are not present in bulk systems. Experimentally, the properties of microgels are accessible by scattering experiments, specifically Neutron Spin Echo (NSE) spectroscopy. ${ }^{10-13}$ Such NSE studies reveal a dynamic crossover from a Zimm-type dynamics to a collective diffusive behavior with a decreasing scattering vector. However, the transition point changes according to the network structure and solvent conditions. $^{9,13}$ On the theoretical side, usually the polymers in the networks are treated as Rouse chains ${ }^{14-16}$ and the

a) Electronic mail: r.winkler@fz-juelich.de network geometries are limited by periodic boundary conditions or simple structures.

In the absence of a well-established theoretical framework, computer simulations are a viable approach to study the internal dynamics of finite-size microgels in solution. Recently, coarse-grained models with an implicit solvent have been successfully employed to investigate the equilibrium properties of neutral and charged microgel systems, ${ }^{17-19}$ but the internal polymer dynamics has not systematically been analyzed, aside from a few studies on core-shell microgels. ${ }^{9}$ The presence of solvent molecules gives rise to long range $\mathrm{HI}$, which has been carefully taken into account in the simulations. There are various mesoscale approaches, which adequately capture HI and can be combined with embedded objects like polymers and colloidal particles. The most widely used approaches are the lattice Boltzmann (LB) method, ${ }^{20-22}$ dissipative particle dynamics (DPD), ${ }^{23,24}$ and the multiparticle collision dynamics (MPC) approach. ${ }^{25-27}$ Here, we adopt the MPC method, a particle-based simulation technique, which incorporates thermal fluctuations, provides hydrodynamic correlations, and is ideally suited to be coupled with molecular dynamics simulations for the microgels. ${ }^{28}$ It has successfully been applied to study equilibrium and nonequilibrium dynamical properties of linear $26,27,29-35$ and star polymers. $^{36-39}$

In this article, we elucidate the internal dynamics of complex microgels by performing mesoscale hydrodynamic simulations. Specifically, we systematically analyze the polymer dynamics of microgels of different swelling degrees, from tightly collapsed to strongly swollen microgels. For this purpose, we determine intermediate scattering functions in 
order to unravel the effect of hydrodynamic interactions and chain stiffness on the polymer dynamics. In addition, the dynamics of individual monomers is studied by means of their mean square displacement (MSD) with respect to the center of mass of the respective polymer and the whole microgel, respectively. The analysis of the MSD is crucial for a correct interpretation of the intermediate scattering data, especially in microgels, which are typically comprised of short polymers (low molecular weight). ${ }^{12}$ Moreover, in order to gain a better understanding of the network dynamics, we also study the intermediate scattering function and the mean square displacement of crosslinks. Our studies are intended to provide insight into the polymer dynamics, which is inaccessible by scattering experiments, e.g., mean square displacements, and to assist in the interpretation of experimental scattering data, e.g., the intermediate scattering function.

The paper is organized as follows. In Section II, the model and simulation approach are outlined. The structure and dynamics of non-charged microgels of various polymer lengths under good solvent condition are studied in Section III A. In Section III B, microgels collapsed in poor solvent conditions are considered. The dynamics of microgels with Debye-Hückel interactions are investigated in Section III C. The crosslink dynamics of collapsed and swollen microgels is studied in Section III D, and Section IV summarizes our results.

\section{MODEL AND METHODS}

\section{A. Microgel model}

A microgel is modeled as a network of tetra-functionally crosslinked polymers, each of length $N_{m}$. Their monomers are treated as point particles of mass $M$, which are connected with their neighboring monomers by the harmonic potential

$$
U_{i, i+1}^{\mathrm{b}}=\frac{K}{2}\left(\left|\boldsymbol{R}_{i+1}-\boldsymbol{R}_{i}\right|-l\right)^{2},
$$

where $\boldsymbol{R}_{i}\left(i=1, \ldots, N_{m}\right)$ is the position of monomer $i, l$ is the bond length, and $K$ is the spring constant. Non-bonded interactions are described by the truncated and shifted Lennard-Jones (LJ) potential

$$
U_{\mathrm{LJ}}=\left\{\begin{array}{rl}
4 \epsilon\left[\left(\frac{\sigma}{R_{i j}}\right)^{12}-\left(\frac{\sigma}{R_{i j}}\right)^{6}\right]-C, & R_{i j}<r_{c} \\
0, & R_{i j}>r_{c}
\end{array},\right.
$$

where $\epsilon$ is the strength of the interaction, $R_{i j}=\left|\boldsymbol{R}_{i}-\boldsymbol{R}_{j}\right|, \sigma$ indicates the diameter of the monomer, $r_{c}$ is the cutoff distance, and $C=4 \epsilon\left[\left(\sigma / r_{c}\right)^{12}-\left(\sigma / r_{c}\right)^{6}\right]$. In order to simulate good solvent conditions, the cutoff distance is set to $r_{c}=2^{1 / 6} \sigma$ and $\epsilon / k_{B} T=1$, where $k_{B}$ is the Boltzmann constant and $T$ is the temperature; in the following, we will denote this as a self-avoiding microgel. For poor solvent conditions, the cutoff distance is set to $r_{c}=2.5 \sigma$ and interaction strengths in the range $0.3 \leq \epsilon / k_{B} T \leq 1.0$ are considered. ${ }^{40}$ This covers a wide range of solvent qualities from nearly good to poor solvent conditions. Swelling by electrostatic interactions is described by the Debye-Hückel potential

$$
U_{\mathrm{DH}}=\left\{\begin{array}{cc}
\frac{k_{\mathrm{B}} T l_{\mathrm{B}}}{R_{i j}} \exp \left(-\frac{R_{i j}}{L_{D}}\right), & R_{i j}<r_{\mathrm{DH}} \\
0, & R_{i j}>r_{\mathrm{DH}}
\end{array}\right.
$$

taking the screening effect of the counterion into account implicitly by the Debye length $L_{\mathrm{D}}$. Here, $l_{B}$ is the Bjerrum length and the cutoff radius is set as $r_{\mathrm{DH}}=5.3 l_{D} \cdot{ }^{18}$

The dynamics of the monomers is treated by molecular dynamics (MD) simulations applying the velocity Verlet integration scheme.

\section{B. Multiparticle collision dynamics fluid}

In the MPC approach, the solvent is represented by $N$ point particles of mass $m$, which are homogeneously spread over a periodic cubic box of length $L$. The dynamics of the solvent particles consist of a streaming step followed by a collision step. ${ }^{26,27}$ During streaming, the particles move ballistically for the time interval $h$ according to

$$
\boldsymbol{r}_{i}(t+h)=\boldsymbol{r}_{i}(t)+h \boldsymbol{v}_{i}(t)
$$

where $\boldsymbol{r}_{i}$ and $\boldsymbol{v}_{i}$ are the position and velocity of particle $i$, $i=1, \ldots, N$. In the collision step, the particles are first sorted in cubic collision cells of length $a^{26,27}$ to determine the interaction environment. Then, for each collision cell, the relative velocities of the particles, with respect to the center-of-massvelocity $\boldsymbol{v}_{\mathrm{cm}}$ of all particles in that cell, are rotated by a fixed angle $\bar{\alpha}$ around a randomly orientate axis. Hence, the velocities of the particles are updated according to

$$
\boldsymbol{v}_{i}(t+h)=\boldsymbol{v}_{\mathrm{cm}}(t)+\mathbf{R}(\bar{\alpha})\left(\boldsymbol{v}_{i}(t)-\boldsymbol{v}_{\mathrm{cm}}(t)\right),
$$

where $\mathbf{R}(\bar{\alpha})$ is the rotation matrix. ${ }^{26,27}$ To guarantee Galilean invariance, a random shift of the entire collision grid is applied at each collision step. ${ }^{41}$

The coupling between the monomers and the MPC solvent is achieved by the inclusion of the monomers in the collision step as described in Refs. 34, 42, and 43.

\section{MPC parameters}

We consider microgel particles of various polymer lengths with a fixed number of crosslinks $N_{c}=729$, which corresponds to 1236 polymers with 64 dangling polymers at the outer surface of the microgel. By choosing $l, k_{\mathrm{B}} T$, and $m$ as units of length, energy, and mass, the unit of time is $\tau=\left(m l^{2} / k_{\mathrm{B}} T\right)^{0.5}$. For the polymers, we set $\sigma=0.8 l, l=a, M=10 m, l_{B}=5 l$, and $K=10^{3} k_{\mathrm{B}} T / l^{2}$, and perform $20 \mathrm{MD}$ simulation steps between collision steps. The parameters of the MPC solvent are chosen as $h=0.1 \tau, \bar{\alpha}=130^{\circ}$, and the average fluid particle number $\left\langle N_{c}\right\rangle=10$. The simulation box size $L$ is set to twice the radius of gyration of the microgels and after equilibrating the structures, we perform at least $5 \times 10^{6} \mathrm{MPC}$ steps in order to achieve an acceptable statistical accuracy.

\section{Analyzing simulation data}

The static structure factor is calculated according to

$$
S(q)=\frac{1}{N} \sum_{i, j=1}^{N}\left\langle\frac{\sin \left(q R_{i j}\right)}{q R_{i j}}\right\rangle
$$


using the simulated trajectories, where $q$ is the magnitude of the scattering vector. The intermediate scattering function is determined by the expression

$$
S(q, t)=\frac{1}{N} \sum_{i, j=1}^{N}\left\langle\exp \left[i \boldsymbol{q} \cdot\left(\boldsymbol{R}_{j}(t)-\boldsymbol{R}_{i}(0)\right)\right]\right\rangle
$$

In order to calculate $S(q, t)$ with reasonable accuracy, the simulation data are stored at every 10 time units and the scattering profiles are calculated via the method proposed by Ramirez et al. $^{44}$

Moreover, we characterize the time dependence of the intermediate scattering function by describing it via the stretched exponential function ${ }^{13}$

$$
S(q, t) / S(q, 0)=\exp \left(-\left[\Gamma_{q} t\right]^{\beta}\right) .
$$

Through fitting, we obtain a decay rate $\Gamma_{q}$ and an exponent $\beta{ }^{45}$ The time interval of the fitting is chosen such that the normalized intermediate scattering profile is larger than $e^{-1}$.

To further characterize the dynamics, we consider the mean square displacement (MSD) of the center of mass of the polymers and the MSD of the monomers with respect to the center of mass of the microgel particle as well as the MSD of the monomers with respect to the center of mass of their polymer.

In addition, the correlation function of the end-to-end vector $\boldsymbol{R}_{\mathrm{ee}}$ is calculated according to

$$
C(t)=\frac{\left\langle\boldsymbol{R}_{\mathrm{ee}}(t) \cdot \boldsymbol{R}_{\mathrm{ee}}(0)\right\rangle}{\left\langle\boldsymbol{R}_{\mathrm{ee}}(0) \cdot \boldsymbol{R}_{\mathrm{ee}}(0)\right\rangle} .
$$

The effective relaxation time $\tau_{1}$ of the end-to-end vector correlation function is obtained by a fit of the stretched exponential function $C(t)=\exp \left[-\left(t / \tau_{1}\right)^{b}\right]$ to the numerically determined correlation functions.

\section{RESULTS AND DISCUSSIONS}

The structural properties of a microgel particle strongly depend on the environmental conditions and, thus, on interactions between the monomers, as illustrated in Fig. 1. In the following, we investigate the effect of microgel swelling on the dynamics of polymers comprising the microgel network.

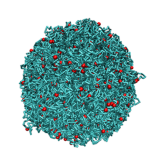

Collapsed

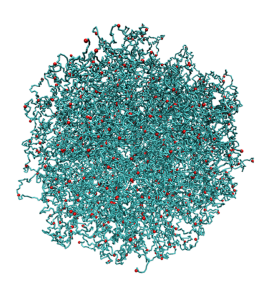

Neutral

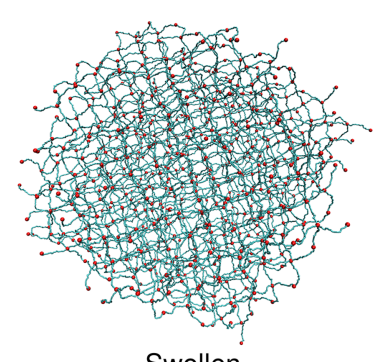

Swollen
FIG. 1. Snapshots of microgels for various monomer interactions. Under bad solvent conditions, the microgel is collapsed ( $\epsilon=1.0$, left), under good solvent conditions, it is neutral (middle), and with repulsive charge interactions, it is swollen $\left(l_{\mathrm{D}}=2.8\right.$, right). Here, $N_{m}=20, N_{c}=729$, and the red dots represent the crosslinks.

\section{A. Microgel under good solvent conditions}

\section{Structure factor}

The static structure factor $S(q)$ of individual polymers under good solvent conditions is presented in Fig. 2 for various polymer lengths. As is well known, we find a shift of the curves for $q l<1$ to smaller $q$ values with the increasing polymer length, which is related to the increasing size of the microgel particle. The polymer specific aspects appear for sufficiently large $q$, where $S(q)$ exhibits a power-law decay with the exponent $-1 / v ; v$ being the Flory exponent. A fit yields the critical exponent $v \approx 0.62$, in agreement with previous simulations of individual polymers of these lengths. ${ }^{29}$ The scaling regime for microgels with longer polymers starts at smaller $q$ values, which can be attributed to the larger end-to-end distance (or larger mesh size) of the individual polymers. Hence, the individual polymers exhibit scaling properties of flexible polymers even in the crosslinked state.

\section{Correlation function}

Figure 3 displays the end-to-end vector correlation function (Eq. (9)) for various polymer lengths. Evidently, $C(t)$ decays in a non-exponential manner, which reflects the contribution of several polymer-internal modes to the correlation function. The polymer-length dependence of the longest polymer relaxation time is presented in the inset of Fig. 3. According to the Zimm model, the longest relaxation time of the polymers scales as $\tau_{1} \sim N^{3 v}$. ${ }^{45}$ With $v=0.62$, this yields the dependence $\tau_{1} \sim N^{1.86}$. The relaxation time of the free polymers shows a $\tau_{1} \sim N^{1.75}$ dependence, i.e., the exponent is somewhat smaller than the theoretical prediction, which might be attributed to the shortness of the considered polymers. The relaxation times obtained for the self-avoiding microgels exhibit the dependence $\tau_{1} \sim N^{1.4}$, which is markedly lower than the theoretical prediction. This small exponent is certainly specific for the considered networks and cannot be explained by the lack or presence of hydrodynamics. To the best of our knowledge, there is no theoretical explanation for this observation yet.

\section{Mean square displacement}

More insights into the monomer dynamics are gained by studying their mean square displacement (cf. Fig. 4). The total mean-square displacement averaged over all monomers

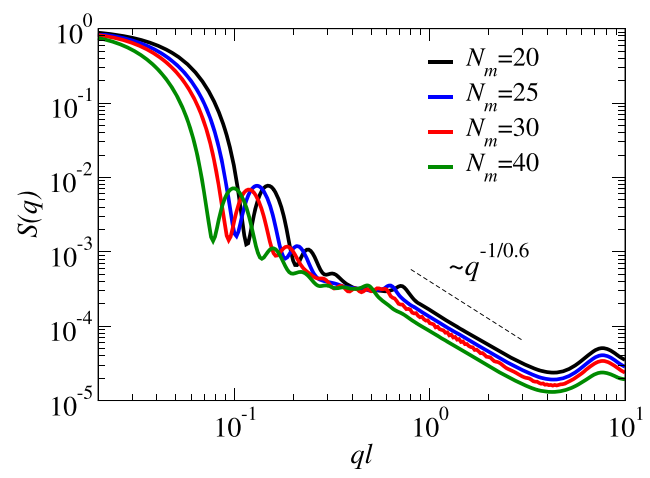

FIG. 2. Static structure factor of self-avoiding microgels for the polymer lengths $N_{m}=20,25,30$, and 40. The dashed line corresponds to $\sim q^{-1 / 0.6}$. 


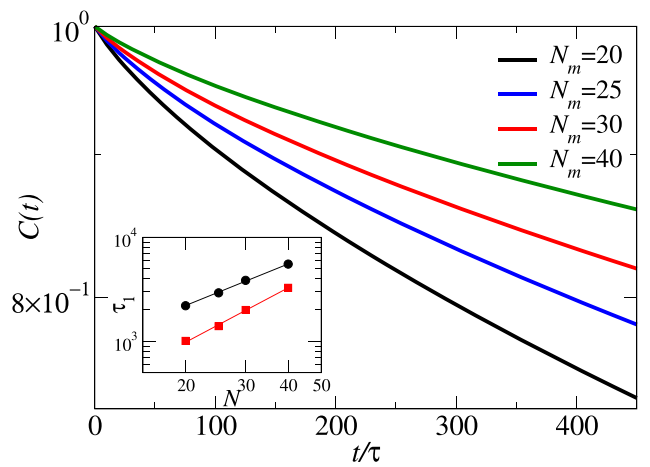

FIG. 3. Correlation function of the end-to-end vector of individual polymers in the microgel network for various polymer lengths $N_{m}$. The inset shows the length dependence of the relaxation time for polymers in the microgel (black circles) and free polymers in dilute solution (red squares).

(solid lines) can be written as the sum of the mean square displacement of the center-of-mass of the individual polymers (dashed-dotted lines) and mean square displacement of the monomers with respect to the center-of-mass of each polymer (dashed lines). The average mean square displacement of the center-of-mass position of the polymers exhibits a diffusive dynamics and increases linearly in time over a large time range. However, the diffusive regime slows down on large time scales due to the hindered movement of the polymers in the microgel network and the diffusive dynamics of the whole microgel is approached. Zimm theory predicts a scaling regime, where the mean-square displacement of the monomers with respect to the polymer center-of-mass increases as $t^{2 / 3} \cdot{ }^{45}$ We do not find a clear and long time range, where this scaling regime applies. We observe a very short time window only, i.e., $20 \leq t /\left(m a^{2} / k_{B} T\right)^{0.5} \leq 70$. This is clearly a consequence of the shortness of the considered polymers and agrees with simulations of polymers in dilute solution. ${ }^{29}$

\section{Intermediate scattering function}

Normalized intermediate scattering functions of polymers of length $N_{m}=20$ in a self-avoiding microgel are shown in Fig. 5(a) for various $q$ values. The functions evidently decay in a non-exponential manner and are well described by Eq. (8).

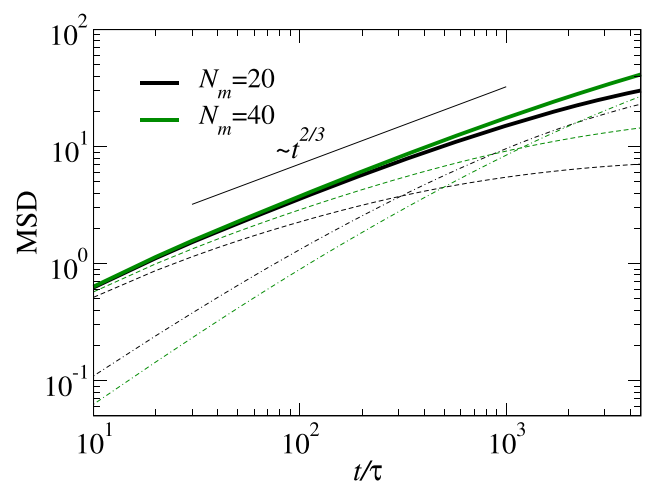

FIG. 4. Monomer mean-square displacements for microgels with $N_{m}=20$ and 40 as a function of time. The solid lines show the averaged monomer mean-square displacement with respect to the center of mass of the microgel. The dashed lines depict the mean-square displacement of the monomers with respect to the center of mass of the polymer where the monomers belong to. The dashed-dotted lines represent the mean-square displacements of the center of mass of each polymer with respect to the center of mass of the microgel.
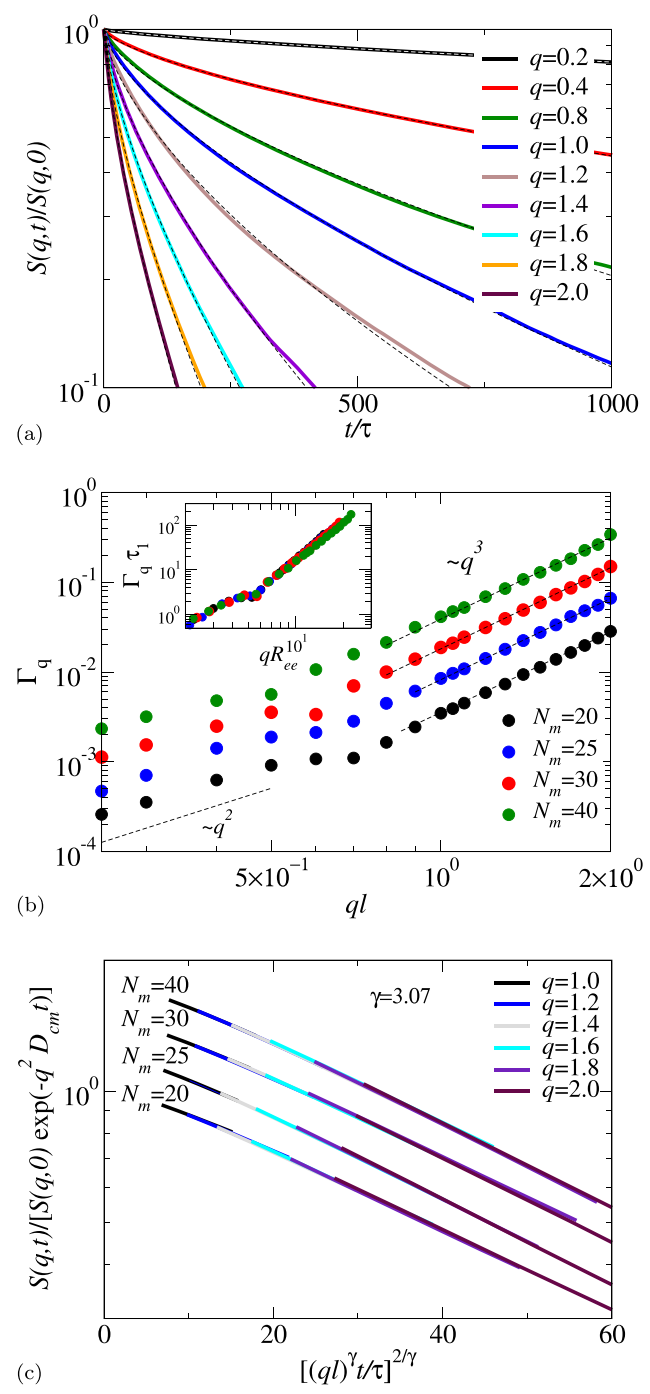

FIG. 5. (a) Intermediate scattering functions for various $q$ values ranging from $q l=0.2$ to 2.0 for polymers in a self-avoiding microgel with $N_{m}=20$. The dashed lines show the fitted stretched exponential functions. (b) Decay rates $\Gamma_{q}$ obtained by fitting the stretched exponential function Eq. (8) to the data. The inset shows scaled decay rates as a function of the normalized magnitude of the scattering vector, $q R_{e e}$. (c) Normalized intermediate scattering functions of microgels of various polymer lengths as a function of scaled time for microgels in a good solvent. Results are presented for $1.0<q l<2.0$ and the time interval $20<t / \tau<70$. Note that in (b) and (c) the data for the various polymer lengths (except for $N_{m}=20$ ) are shifted vertically for better visibility.

The extracted decay rates are displayed in Fig. 5(b). The Zimm model predicts for these parameters $\Gamma_{q} \sim q^{2}$ and $\beta=1$ as long as $\left(\boldsymbol{q} \boldsymbol{R}_{e e}\right)^{2} \ll 1$, or $\Gamma_{q} \sim q^{3}$ and $\beta=2 / 3$ for $\left(\boldsymbol{q} \boldsymbol{R}_{e e}\right)^{2} \gg 1$ and $t / \tau_{1} \ll 1 \ll \Gamma_{q} t .{ }^{45}$ Our simulation results are in agreement with these predictions. For low $q$ values and all polymer lengths, the decay rates exhibit approximately the power-law dependence $q^{2}$. This is attributed to a collective diffusion-like motion of the polymers inside the microgel network. ${ }^{13}$ For larger $q, \Gamma_{q}$ shows a transition to $\mathrm{a} \sim q^{3}$ regime due to the hydrodynamic interactions in agreement with the theoretical expectations. It is interesting to note that the transition point from $\mathrm{a} \sim q^{2}$ to $\mathrm{a} \sim q^{3}$ regime shifts to larger $q$ values as the polymer length $N_{m}$ decreases. This is consistent with the polymer scaling regime shown in Fig. 2.

In order to exclude the effect of polymer length, the decay rates are multiplied by the relaxation time $\tau_{1}$ and are plotted 
against the normalized scattering vector $q R_{e e}$ (see the inset of Fig. 5(b)). As is evident, all of the decay rates fall on a master curve with two distinct regimes: a diffusive regime characterized by $\Gamma_{q} \sim q^{2}$ at low $q$, i.e., $q R_{e e} / 2 \pi<1$, followed by a $\Gamma_{q} \sim q^{3}$ regime due to hydrodynamic interactions for large $q$, i.e., $q R_{e e} / 2 \pi>1$. For single free polymers, the intermediate scattering function exhibits the universal scaling relation $S(q, t)=S(q, 0) f\left(q^{\gamma} t\right)$ for a suitable range of $q$ and time scales. ${ }^{29,45-47}$ The analytical calculation for a flexible Gaussian polymer and scaling considerations for a polymer with excluded volume interactions in solution (Zimm model) yields an exponential function for $f(x)$, with $x \sim\left(q^{\gamma} t\right)^{2 / \gamma}$ and $\gamma=3{ }^{46-48}$ Polymer stiffness changes the exponent to $\gamma=8 / 3$ for semiflexible polymers. ${ }^{46}$ The structure factor (Fig. 2) and the monomer mean square displacement in the center-of-mass of the polymers (Fig. 4) suggest such a universal scaling regime for $1.0<q l<2.0$ and Zimm dynamics for $20<t / \tau<70$. As displayed in Fig. 5(c), the intermediate scattering curve follows the dynamical scaling relation rather well with $\gamma \approx 3.07$, as for similar polymers in dilute solution. ${ }^{29}$ This suggests that the polymer dynamics in the crosslinked network of the microgels under good solvent conditions is governed by hydrodynamic interactions. Note, in order to see the internal polymer dynamics only, the center-of-mass dynamics is removed by dividing the intermediate scattering function by $\exp \left(-q^{2} D_{\mathrm{cm}} t\right)$, where $D_{\text {cm }}$ is the diffusion coefficient of the center-of-mass motion of the polymers with respect to the center of mass of the microgel.

\section{B. Microgel under poor solvent conditions}

\section{Structure factor}

The equilibrium properties of microgels at different swelling conditions have been studied previously. ${ }^{18}$ Here, we are primarily interested in the dynamics of the polymers in the different swollen states of the microgel. From this point onwards, we consider the microgel in a good solvent with $N_{m}=20$ as reference and discuss microgel swelling and collapse with respect to that. Static structure factors $S(q)$ of microgels of various degrees of swelling, i.e., $\epsilon$ values, are presented in Fig. 6. With increasing $\epsilon$, the $S(q)$ curves shift to large $q$ values, consistent with the decreasing size of the microgels. As long as $\epsilon / k_{B} T \lesssim 0.5$, there is still a scaling regime for $q l>1$. However, the slope of the decaying regime becomes steeper with increasing $\epsilon$ compared to the self-avoiding case.

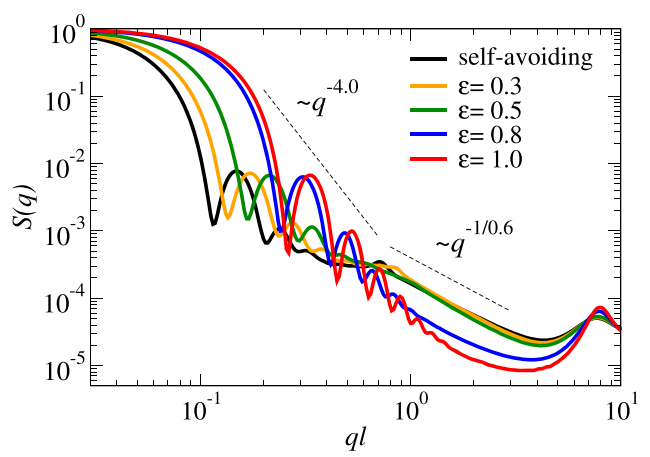

FIG. 6. Static structure factors of microgels under bad solvent conditions for various degrees of swelling. The microgel-size decreases with increasing $\epsilon$.
Monomer-monomer attractions compensate excluded-volume interactions, and the polymer conformations become thetasolvent-like. Yet for even larger values of $\epsilon$, the microgel drastically shrinks and exhibits a compact state with a sharp boundary (cf. Fig. 1). Then the structure factor curves follow a $q^{-4}$ scaling.

\section{Mean square displacement}

The monomer mean square displacement curves for microgels with various attraction strengths are shown in Fig. 7(a). The MSD curves for the partially swollen microgels (i.e., $\epsilon / k_{B} T \leq 0.5$ ) are close to that of the self-avoiding microgel and the average mean square displacement of the monomers in the center-of-mass reference frame of the polymer scales as $\sim t^{2 / 3}$ at short times. For strongly collapsed microgels (i.e., $\epsilon / k_{B} T>0.5$ ), the exponent of the mean square

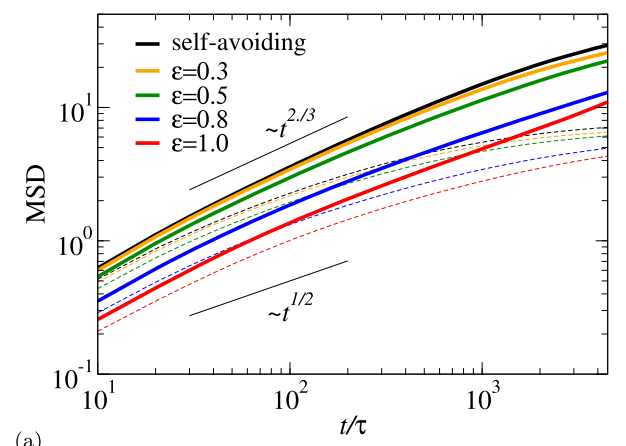

(a)
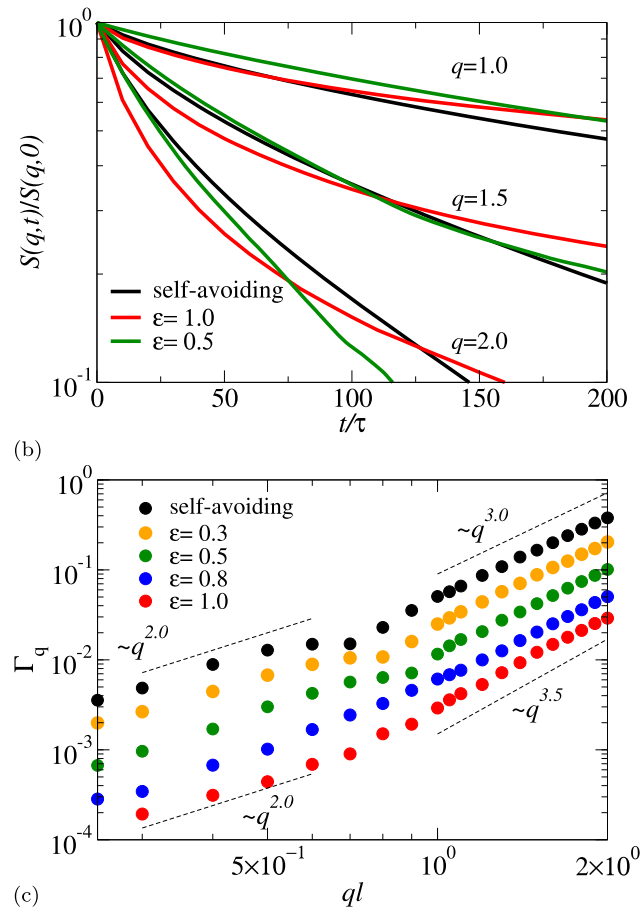

FIG. 7. (a) Average monomer mean-square displacements for collapsed microgels with respect to the center of mass of the microgel. The dashed lines show the monomers mean-square displacement with respect to the center of mass of the polymers. (b) Intermediate scattering functions of polymers for self-avoiding, partially collapsed $\left(\epsilon / k_{B} T=0.5\right)$, and strongly collapsed $\left(\epsilon / k_{B} T=1.0\right)$ microgels for $q l=1.0,1.5$, and 2.0. (c) Decay rates $\Gamma_{q}$ of the intermediate scattering functions for microgels of different degrees of swelling. Note that the plots (except for the self-avoiding microgel) are shifted vertically for better visibility. 
displacement curve decreases and longer times are required to enter the polymer scaling regime.

\section{Decay rate}

The intermediate scattering functions of the polymers in the collapsed microgel exhibit a significantly different time dependence compared to those under good solvent conditions as shown in Fig. 7(b). For strongly collapsed microgels, $S(q, t)$ decays significantly faster at short times compared to the self-avoiding case, whereas a slower decay is observed for long times. The decay rates for the self-avoiding and collapsed microgels are compared in Fig. 7(c). As expected, the results show a crossover from a $q^{2}$ to a $q^{3}$ dependence of $\Gamma_{q}$ for $\epsilon / k_{B} T \leq 0.5$ which is similar to our previous results for a selfavoiding microgel. However, the transition point shifts toward higher values as $\epsilon$ increases. For higher interaction strengths (i.e., $\epsilon / k_{B} T>0.5$ ), we observe no clear transition point and the $q$ dependency of $\Gamma_{q}$ gradually changes from a $q^{2}$ dependence to a regime with exponents larger that $\gamma=3.0$. This is also reflected in the mean square displacement curves of the collapsed microgels (see Fig. 7(a)). For the completely collapsed microgel (i.e., $\epsilon / k_{B} T=1.0$ ), the exponent of the time scaling regime is lower than $2 / 3$ and is closer to $1 / 2$ for $t / \tau>70$. We attribute this lower exponent of the MSD curves and consequently higher scaling exponent of the decay rates $\gamma$ to a screening of hydrodynamic interactions, which is certainly the case for the collapsed microgel, and which ultimately leads to an exponent of $\gamma=4.0$, corresponding to the Rouse model. ${ }^{45}$

\section{Swollen microgels with Debye-Hückel interactions}

\section{Structure factor}

Microgels in the presence of electrostatic interactions (ionic microgels) at low salt concentrations can be significantly more swollen than self-avoiding microgels. ${ }^{49}$ To analyze the effect of such a strong swelling on the internal dynamics of a microgel, we study the polymer dynamics within a DebyeHückel description of the charge interaction. Of course, this neglects correlations between the polymers and counterions but sheds light on differences to less swollen polymers. Again, we consider polymers of length $N_{m}=20$, where the individual monomers interact via the Debye-Hückel potential (Eq. (3)). As shown by the static structure factor in Fig. 8, the chain conformations exhibit a crossover from self-avoiding to rodlike polymer behavior with increasing Debye length $L_{D}$. In the

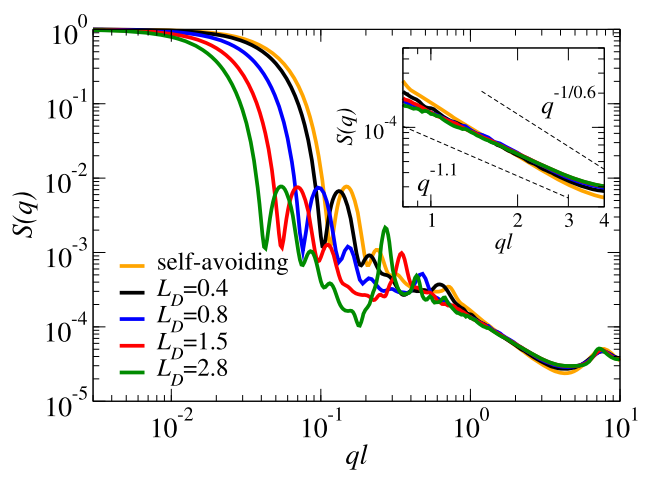

FIG. 8. Static scattering functions for $N_{m}=20, L_{D}=0.4,0.8,1.5$, and 2.8. polymer scaling regime, $S(q)$ is approximately proportional to $q^{-1 / 0.67}$ and $q^{-1.1}$ for $L_{D} / l=0.4$ and 2.8 , respectively, which is consistent with our findings in Ref. 18.

\section{Mean square displacement}

Monomer mean square displacement curves for various Debye lengths are presented in Fig. 9. The results show that the average mean square displacement decreases with increasing $L_{D}$. This is consistent with theoretical expectations for the crossover from flexible to semiflexible and stiff polymers. ${ }^{46}$ Simultaneously, the scaling regime $\left(t^{2 / 3}\right)$ for the mean square displacement of the monomers with respect to the polymer center of mass, which is shortened with increasing $L_{D}$, seems to be shifted to shorter times, and a broader crossover regime to the asymptotic value for $t \rightarrow \infty$ appears. For small $L_{D}$ values, i.e., $L_{D} / l=0.4$ and 0.8 still a time regime over which the monomer dynamics is dominated by hydrodynamic interactions is observed $\left(t^{2 / 3}\right)$, but the interval of the time regime is shorter compared to a self-avoiding microgel. However, for larger Debye lengths, $L_{D} / l=1.5$ and 2.8 , a very short $t^{2 / 3}$ scaling regime is observed as the mean square displacement of the monomers is suppressed due to the shortness of the polymers and hindered movement by the crosslinks. This implies that for large Debye lengths, the monomer mean square displacement is in a crossover regime even for very short times $t / \tau \gtrsim 10$. We like to emphasize that the monomer mean square displacements, with respect to the center of mass of the microgel, for the various persistence lengths exhibit a time dependence close to $t^{2 / 3}$ over a wide time window. One has to bear in mind that this displacement does not reflect the internal polymer dynamics but rather the coupled dynamics of the network. The theoretical predictions in terms of scaling properties are typically not meant for that dynamics.

\section{Decay rate}

The intermediate scattering function for various $q$-values closely resembles that of self-avoiding microgels (cf. Fig. 5(a)). The decay rates $\Gamma_{q}$ for various Debye lengths are compared with those of self-avoiding microgels in Fig. 10(a). The results suggest that as the Debye length increases, the crossover from the collective diffusive regime to non-diffusive

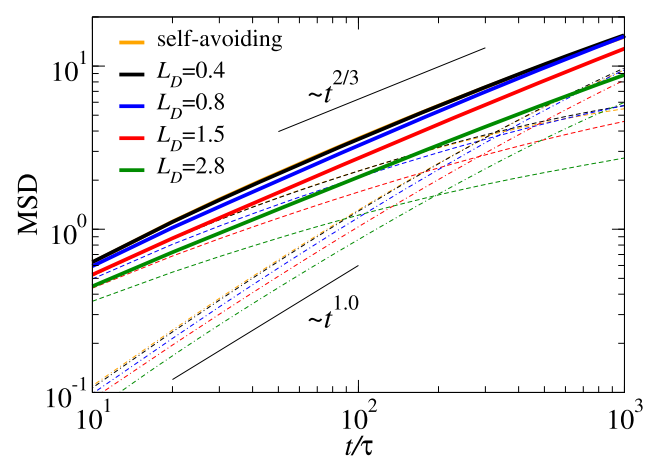

FIG. 9. Average mean square displacement of monomers with respect to the center of mass of the microgel (solid lines) and with respect to the center of mass of the polymer (dashed lines). The mean square displacement of the center of mass of the polymers with respect to the center of mass of the microgel is shown by dotted-dashed lines. Thin black lines are included as guide. 

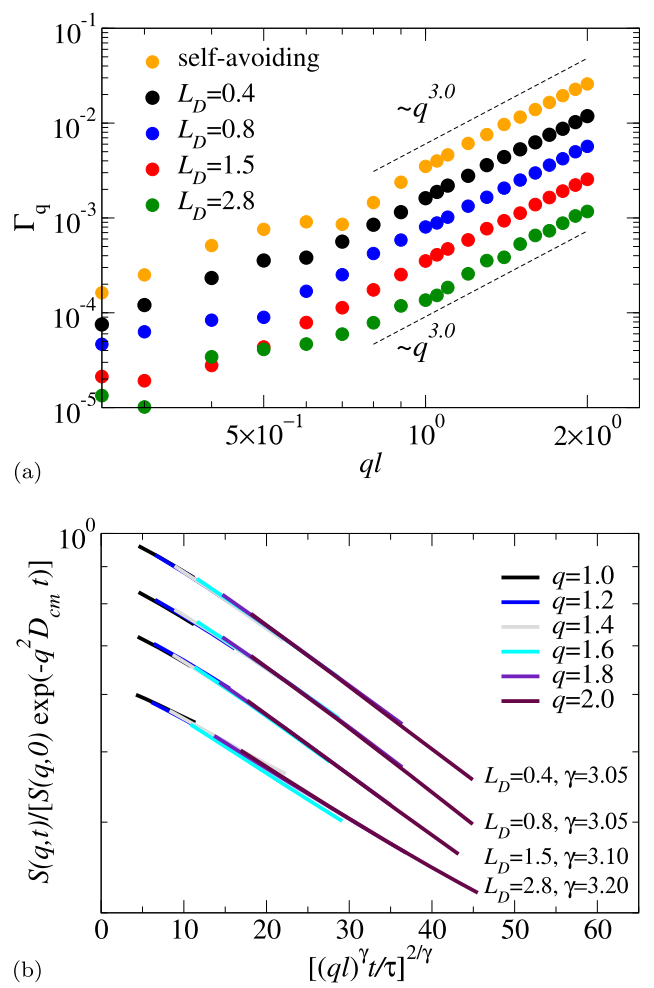

FIG. 10. (a) Decay rates $\Gamma_{q}$ of microgels with $N_{m}=20$ for $L_{D}=0.4,0.8$, 1.5 , and 2.8. The dashed lines are guides for $q^{3}$ and $q^{2.85}$ scaling. Note that the data are shifted vertically for better visibility, except for the self-avoiding microgel. (b) Intermediate scattering profiles as a function of the scaled time for microgels with $N_{m}=20$. Results are presented for $1.0<q l<2.0$ and $10 \leq t / \tau \leq 50$, for $L_{D}=0.4,0.8,1.5$, and 2.8. Note that the scaling curves are shifted vertically (except for $L_{D}=0.4$ ) for better visibility.

regime shifts to lower $q$. This is indeed due to the increased persistence length of the polymers in the presence of long-range repulsive electrostatic interactions. At larger $q$, i.e., $q l>0.8$, the exponent of the $q$-dependence of $\Gamma_{q}$ is approximately 3 for self-avoiding microgels as well as for swollen microgels with Debye-Hückel interactions. This is in disagreement with the theoretical prediction for stiff polymers suggesting a dependence $\Gamma_{q} \sim q^{8 / 3}$ of the decay rate on $q{ }^{46}$ This discrepancy can be explained through the mean square displacement curves shown in Fig. 9. Since the mean square displacement curves for swollen microgels show no $t^{3 / 4}$ behavior due to the shortness of the polymers, no $\Gamma_{q} \sim q^{8 / 3}$ scaling can be expected for the decay rate. The exponent 3.0 for the $q$ dependence of the decay rates is rather consistent with the slope of the mean square displacement of the monomers with respect to the center of mass of the microgels which for all Debye lengths shows approximately a $t^{2 / 3}$ behavior within the fitting time interval (see Fig. 9). Thus, the decay rate does not simply reflect the internal dynamics of the polymers but rather the monomer dynamics in the coupled network.

\section{Intermediate scattering function}

In contrast to the collapsed microgels, no slow decaying regime is observed in the intermediate scattering functions of the swollen microgels and the functional form of the intermediate scattering functions closely resembles those of the self-avoiding microgels. In Fig. 10(b), the universal scaling properties of the intermediate scattering profiles for swollen microgels are illustrated for the interval $1.0<q l<2.0$ and the time range $10 \leq t / \tau \leq 50$. It should be noted that the effect of the center-of-mass movement of the polymers is excluded by dividing the intermediate scattering functions by $\exp \left(-q^{2} D_{\mathrm{cm}} t\right)$. The result suggests that the polymer dynamics follows the universal scaling relation $S(q, t)=S(q, 0) f\left(q^{\gamma} t\right) / \exp \left(-q^{2} D_{c m} t\right)$ quit well. The scaling exponent is close to $\gamma=3$ for $L_{D} / l=0.4$ and 0.8 . For the larger persistence lengths, the exponent increases to $\gamma=3.1\left(L_{D} / l\right.$ $=1.5)$ and $\gamma=3.2\left(L_{D} / l=2.8\right)$. Although this contradicts the expectation for stiff semiflexible polymers, it is consistent with the behavior of the mean square displacement curves showing a slope smaller than 2/3 (see Fig. 9) for larger Debye lengths in the fitting time interval. Hence, we still observe a scaling behavior for the intramolecular dynamics of the polymers, but with scaling exponents differing from theoretical predictions. This is also observed in recent experimental studies on cationic microgels where the polymer dynamics can be better explained by the Zimm model rather than a semiflexible polymer. ${ }^{50}$ Nevertheless, the polymer dynamics is determined by hydrodynamic interactions, which we conclude from the small deviations of $\gamma$ from 3 .

\section{Crosslink dynamics}

\section{Mean square displacement}

The internal dynamics of the self-avoiding, collapsed, and swollen microgels is further analyzed by the mean square displacement of the crosslinks (see Fig. 11). The MSD curves for the various degrees of the microgel swelling exhibit distinctively different power-law exponents. For the most strongly collapsed microgel, we find a crossover from an approximate $t^{3 / 4}$ dependence $\left(20<t / \sqrt{m a^{2} / k_{\mathrm{B}} T}<70\right)$ to $\mathrm{a} \sim t^{1 / 2}$ regime for longer times. The crossover time seems to shift to longer times with an increasing degree of microgel swelling, such that the strongest swollen systems exhibit the dependence $t^{3 / 4}$ over the whole considered time window. Hence, the crosslinks exhibit a distinctively different dynamics compared to the monomer dynamics. The coupling between the various polymers yields a dynamics typical for semiflexible polymers at short times for collapsed microgels and a larger time-range for swollen microgels. The collapsed microgels show a long-time behavior typical for Rouse polymers without hydrodynamic interactions.

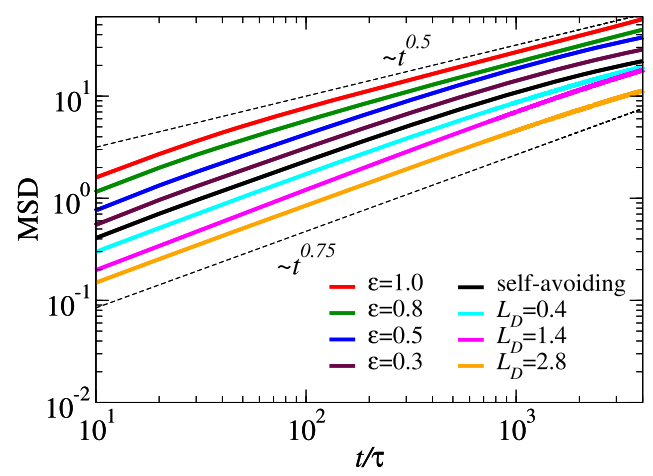

FIG. 11. Mean square displacement of crosslinks in collapsed and swollen microgels. Note that the plots are shifted vertically (except self-avoiding microgel) for better visibility. 


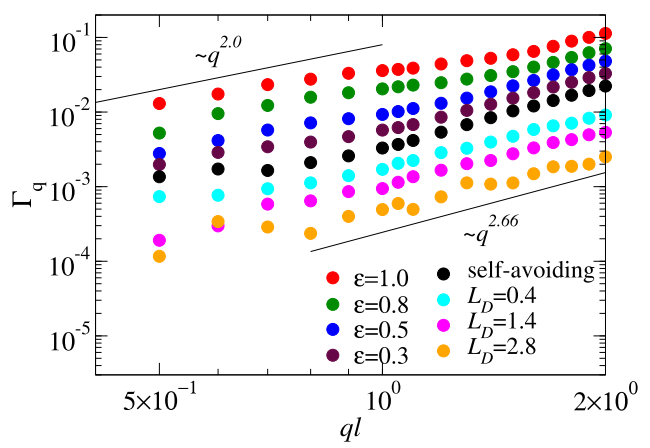

FIG. 12. Decay rates $\Gamma_{q}$ of crosslinks intermediate scattering profiles for microgels with different swelling degrees. Note that the plots are shifted vertically (except self-avoiding microgel) for better visibility.

Figure 12 displays the decay rate of the intermediate scattering function of the crosslinks. At large $q$-values, we expect that $S(q, t)$ reflects the dynamics of nearby crosslinks. Assuming a negligible diffusion constant of a microgel, the intermediate scattering profile for crosslinks can be written as $S(q, t) \sim S(q, 0) \exp \left(-q^{2}\left\langle\Delta r_{\mathrm{cl}}(t)^{2}\right\rangle / 6\right)$, where $\left\langle\Delta r_{\mathrm{cl}}(t)^{2}\right\rangle$ is the mean-squared displacement of a crosslink. Therefore, the decay rate scales as $\Gamma_{q} \sim q^{2 / \alpha}$, with $\alpha$ being the exponent of the mean-square displacement curve. The results in Fig. 12 show that $\Gamma_{q}$ for crosslinks indeed exhibits an approximate $q^{8 / 3}$ scaling at sufficiently high $q$, which is compatible with the $t^{3 / 4}$ behavior of the crosslinks shown in Fig. 11 .

The dynamics of the crosslinks is evidently significantly different from the monomer dynamics. In order to understand the different regimes of the crosslink dynamics better, an analytical description is needed. Specifically, the underlying mechanisms leading to semiflexible polymer features need to be unraveled.

\section{SUMMARY AND CONCLUSIONS}

We have exploited a hybrid mesoscale hydrodynamic simulation approach to study the internal dynamics of microgel particles. The method combines multiparticle collision dynamics simulations for the fluid with molecular dynamics simulations for the microgel. For these simulations, a simplified representation of microgel topology is considered, where polymers are arranged in a regular network with polymer ends being tetra-functionally crosslinked.

A wide spectrum of microgel structures has been considered, from strongly collapsed to completely swollen. This includes self-avoiding microgels corresponding to good solvent conditions, where the conformations are determined by excluded-volume interactions, entropy, and network topology. "Poor solvent" conditions, where hydrophobic interactions dominate, are achieved by an attractive LJ interaction characterized by the depth $\epsilon$ of the potential. The "swollen" state is described by introducing Debye-Hückel interactions between charged monomers, where the strength of charge repulsion is defined by the Debye length $L_{D}$.

The polymer conformations were first characterized by the static structure factor, where we observed the expected change in the scaling behavior in terms of $q$ from a selfavoiding polymer $\left(S(q) \sim q^{-1 / v}\right.$, with $\left.v \approx 0.62\right)$ to a larger exponent for a collapsed microgel. For the swollen microgels, a smaller exponent was observed similar to that for a stretched polymer $\left(S(q) \sim q^{-1.1}\right)$ which closely resembles a rod-like behavior.

The dynamical behavior is less clearly distinguishable, which is mainly a consequence of the shortness of the polymers. For various polymer lengths of self-avoiding microgels, we observe a crossover from the collective diffusive-like dynamics to Zimm scaling for the decay rate of the intermediate scattering function as $q$ increases. This Zimm-type scaling is also reflected in the scattering function itself, suggesting that the polymer dynamics is mainly determined by hydrodynamic interactions in the self-avoiding microgels. This also applies to partially collapsed and weakly swollen microgels. However, strongly collapsed microgels exhibit a decay rate dependence of $\Gamma_{q} \sim q^{3.5}$, with an exponent larger than the Zimm value 3.0. We attribute this to the (partial) screening of hydrodynamic interactions.

Interestingly, the decay rate of swollen microgels does not reflect the stretching of the polymers as is evident in the structure factor. This unexpected behavior is traced back to the lack of a semiflexible-polymer-like diffusive behavior in the monomer mean square displacement. The monomer MSD shows a rather broad crossover behavior which starts from very short times due to the shortness of the polymers. Therefore, the intramolecular polymer dynamics in the studied microgels does not show signatures which are typical and are most pronounced for long polymers. ${ }^{45}$ It should be noted that the decay rate is determined from the intermediate scattering function comprising the intramolecular and center-of-mass dynamics of a polymer. Here, the monomer MSD shows a consistent seemingly hydrodynamics-dominated time dependence which is merely a consequence of the superposition of the center-of-mass and intramolecular dynamics. Hence, the interpretation of dynamical measurements in terms of universal polymer scaling relations must be performed with care, since the polymers in microgels are typically short.

Furthermore, we have analyzed the dynamics of the crosslinks. This is relevant since it could be measured experimentally by suitably labeling the crosslinkers. Interestingly, the crosslink MSD and decay rate of the intermediate scattering function exhibit features corresponding to semiflexible polymer properties. According to our results, this behavior cannot be simply explained through available theoretical frameworks for single free polymers and unraveling the underlying dynamics demands an appropriate analytical model which is out of the scope of the current manuscript.

While most of the research works are focused on the structure of microgel particles, to the best of our knowledge, this is the first work systematically addressing the internal polymer dynamics in responsive microgels. Here, we made an effort to characterize the dynamical features of polymers in microgels under different swelling conditions and where possible examined them against available theoretical predictions. The complex dynamics of polymers in the microgel network is the result of several overlapping processes which are not easily separable. These include limited motion of polymers in the crosslinked network of the microgel, hindered movement of monomers with respect to the center of mass of 
the polymers, and short relaxation time of the polymers due the short polymer lengths. We hope that our results will be helpful in experimental studies of the polymer dynamics in microgels and serve as a guide in the interpretation of scattering results. Moreover, modifications of scattering results due to impurities might be resolved by comparison with our investigations.

\section{ACKNOWLEDGMENTS}

This research work was supported by the Deutsche Forschungs Gemeinschaft within the Sonderforschungsbereich (No. SFB 985) "Functional Microgels and Microgel Systems."

${ }^{1}$ R. Pelton and P. Chibante, "Preparation of aqueous latices with N-isopropylacrylamide," Colloids Surf. 20, 247-256 (1986).

${ }^{2}$ R. Pelton, "Temperature-sensitive aqueous microgels," Adv. Colloid Interface Sci. 85, 1-33 (2000).

${ }^{3}$ N. Murthy, M. Xu, S. Schuck, J. Kunisawa, N. Shastri, and J. M. Fréchet, "A macromolecular delivery vehicle for protein-based vaccines: Aciddegradable protein-loaded microgels," Proc. Natl. Acad. Sci. U. S. A. 100, 4995-5000 (2003).

${ }^{4}$ V. C. Lopez and M. Snowden, "The role of colloidal microgels in drug delivery," Drug Delivery Syst. Sci. 3, 19-23 (2003).

${ }^{5}$ G. Morris, B. Vincent, and M. Snowden, "Adsorption of lead ions onto $\mathrm{N}$-isopropylacrylamide and acrylic copolymer microgels," Prog. Colloid Polym. Sci. 105, 16 (1997).

${ }^{6}$ S. Xu, J. Zhang, C. Paquet, Y. Lin, and E. Kumacheva, "From hybrid microgels to photonic crystals," Adv. Funct. Mater. 13, 468-472 (2003).

${ }^{7}$ L. Bromberg, M. Temchenko, and T. A. Hatton, "Smart microgel studies. Polyelectrolyte and drug-absorbing properties of microgels from polyethermodified poly (acrylic acid)," Langmuir 19, 8675-8684 (2003).

${ }^{8}$ J. Zhang, S. Xu, and E. Kumacheva, "Polymer microgels: Reactors for semiconductor, metal, and magnetic nanoparticles," J. Am. Chem. Soc. 126, 7908-7914 (2004).

${ }^{9}$ S. Maccarrone, A. Ghavami, O. Holderer, C. Scherzinger, P. Lindner, W. Richtering, D. Richter, and R. G. Winkler, "Dynamic structure factor of core-shell microgels: A neutron scattering and mesoscale hydrodynamic simulation study," Macromolecules 49, 3608-3618 (2016).

${ }^{10}$ A. Fernandez-Barbero, A. Fernandez-Nieves, I. Grillo, and E. LopezCabarcos, "Structural modifications in the swelling of inhomogeneous microgels by light and neutron scattering," Phys. Rev. E 66, 051803 (2002).

${ }^{11}$ C. Scherzinger, P. Lindner, M. Keerl, and W. Richtering, "Cononsolvency of poly ( $N, N$-diethylacrylamide) (PDEAAM) and poly $(N$-isopropylacrylamide) (PNIPAM) based microgels in water/methanol mixtures: Copolymer vs core- shell microgel," Macromolecules 43, 6829-6833 (2010).

${ }^{12}$ C. Scherzinger, O. Holderer, D. Richter, and W. Richtering, "Polymer dynamics in responsive microgels: Influence of cononsolvency and microgel architecture," Phys. Chem. Chem. Phys. 14, 2762-2768 (2012).

${ }^{13}$ S. Maccarrone, C. Scherzinger, O. Holderer, P. Lindner, M. Sharp, W. Richtering, and D. Richter, "Cononsolvency effects on the structure and dynamics of microgels," Macromolecules 47, 5982-5988 (2014).

${ }^{14}$ V. P. Toshchevikov, A. Blumen, and Y. Y. Gotlib, "Dynamics of polymer networks with strong differences in the viscous characteristics of their crosslinks and strands," Macromol. Theory Simul. 16, 359-377 (2007).

${ }^{15}$ A. A. Gurtovenko and Y. Y. Gotlib, "Intra-and interchain relaxation processes in meshlike polymer networks," Macromolecules 31, 5756-5770 (1998).

${ }^{16}$ A. A. Gurtovenko and Y. Y. Gotlib, "Viscoelastic dynamic properties of meshlike polymer networks: Contributions of intra-and interchain relaxation processes," Macromolecules 33, 6578-6587 (2000).

${ }^{17}$ M. Quesada-Perez and A. Martin-Molina, "Monte Carlo simulation of thermo-responsive charged nanogels in salt-free solutions," Soft Matter $\mathbf{9}$, 7086 (2013).

${ }^{18} \mathrm{H}$. Kobayashi and R. G. Winkler, "Structure of microgels with Debye-Hückel interactions," Polymers 6, 1602-1617 (2014).

${ }^{19}$ A. M. Rumyantsev, A. A. Rudov, and I. I. Potemkin, "Communication: Intraparticle segregation of structurally homogeneous polyelectrolyte microgels caused by long-range coulomb repulsion,” J. Chem. Phys. 142, 171105 (2015).

${ }^{20}$ G. R. McNamara and G. Zanetti, "Use of the Boltzmann equation to simulate lattice-gas automata," Phys. Rev. Lett. 61, 2332 (1988).

${ }^{21} \mathrm{X}$. Shan and H. Chen, "Lattice Boltzmann model for simulating flows with multiple phases and components," Phys. Rev. E 47, 1815 (1993).

${ }^{22}$ X. He and L.-S. Luo, "Theory of the lattice Boltzmann method: From the Boltzmann equation to the lattice Boltzmann equation," Phys. Rev. E 56, 6811 (1997).

${ }^{23}$ P. J. Hoogerbrugge and J. M. V. A. Koelman, "Simulating microscopic hydrodynamics phenomena with dissipative particle dynamics," Europhys. Lett. 19, 155 (1992)

${ }^{24}$ P. Español and P. B. Warren, "Statistical mechanics of dissipative particle dynamics," Europhys. Lett. 30, 191 (1995).

${ }^{25}$ A. Malevanets and R. Kapral, "Mesoscopic model for solvent dynamics," J. Chem. Phys. 110, 8605 (1999).

${ }^{26} \mathrm{R}$. Kapral, "Multiparticle collision dynamics: Simulations of complex systems on mesoscale," Adv. Chem. Phys. 140, 89 (2008).

${ }^{27}$ G. Gompper, T. Ihle, D. M. Kroll, and R. G. Winkler, "Multi-particle collision dynamics: A particle-based mesoscale simulation approach to the hydrodynamics of complex fluids," Adv. Polym. Sci. 221, 1 (2009).

${ }^{28}$ C.-C. Huang, G. Gompper, and R. G. Winkler, "Hydrodynamic correlations in multiparticle collision dynamics fluids," Phys. Rev. E 86, 056711 (2012).

${ }^{29}$ K. Mussawisade, M. Ripoll, R. G. Winkler, and G. Gompper, "Dynamics of polymers in a particle based mesoscopic solvent," J. Chem. Phys. 123, 144905 (2005).

${ }^{30}$ J. F. Ryder and J. M. Yeomans, "Shear thinning in dilute polymer solutions," J. Chem. Phys. 125, 194906 (2006).

${ }^{31}$ S. Frank and R. G. Winkler, "Polyelectrolyte electrophoresis: Field effects and hydrodynamic interactions," EPL 83, 38004 (2008).

${ }^{32}$ R. Chelakkot, R. G. Winkler, and G. Gompper, "Flow-induced helical coiling of semiflexible polymers in structured microchannels," Phys. Rev. Lett. 109, 178101 (2012).

${ }^{33}$ A. Nikoubashman and C. N. Likos, "Flow-induced polymer translocation through narrow and patterned channels," J. Chem. Phys. 133, 074901 (2010).

${ }^{34}$ C.-C. Huang, R. G. Winkler, G. Sutmann, and G. Gompper, "Semidilute polymer solutions at equilibrium and under shear flow," Macromolecules 43, 10107 (2010).

${ }^{35}$ C. C. Huang, G. Gompper, and R. G. Winkler, "Effect of hydrodynamic correlations on the dynamics of polymers in dilute solution," J. Chem. Phys. 138, 144902 (2013).

${ }^{36}$ M. Ripoll, R. G. Winkler, and G. Gompper, "Star polymers in shear flow," Phys. Rev. Lett. 96, 188302 (2006).

${ }^{37}$ D. A. Fedosov, S. P. Singh, A. Chatterji, R. G. Winkler, and G. Gompper, "Semidilute solutions of ultra-soft colloids under shear flow," Soft Matter 8, 4109 (2012).

${ }^{38}$ S. P. Singh, C.-C. Huang, E. Westphal, G. Gompper, and R. G. Winkler, "Hydrodynamic correlations and diffusion coefficient of star polymers in solution," J. Chem. Phys. 141, 084901 (2014).

${ }^{39}$ R. G. Winkler, D. A. Fedosov, and G. Gompper, "Dynamical and rheological properties of soft colloid suspensions," Curr. Opin. Colloid Interface Sci. 19, 594 (2014).

${ }^{40}$ G. Luna-Bárcenas, J. C. Meredith, I. C. Sanchez, K. P. Johnston, D. G. Goromov, and J. J. de Pablo, "Relationship between polymer chain conformation and phase boundaries in a supercritical fluid," J. Chem. Phys. 107, 10782-10792 (1997).

${ }^{41}$ T. Ihle and D. Kroll, "Stochastic rotation dynamics: A Galilean-invariant mesoscopic model for fluid flow," Phys. Rev. E 63, 020201 (2001).

${ }^{42} \mathrm{~A}$. Malevanets and J. M. Yeomans, "Dynamics of short polymer chains in solution," Europhys. Lett. 52, 231-237 (2000).

${ }^{43}$ M. Ripoll, K. Mussawisade, R. G. Winkler, and G. Gompper, "Low-Reynolds-number hydrodynamics of complex fluids by multiparticle-collision dynamics," Europhys. Lett. 68, 106 (2004).

${ }^{44}$ J. Ramirez, S. K. Sukumaran, B. Vorselaars, and A. E. Likhtman, "Efficient on the fly calculation of time correlation functions in computer simulations," J. Chem. Phys. 133, 154103 (2010).

${ }^{45}$ M. Doi and S. F. Edwards, The Theory of Polymer Dynamics (Clarendon Press, Oxford, 1986).

${ }^{46}$ L. Harnau, R. G. Winkler, and P. Reineker, "Dynamic structure factor of semiflexible macromolecules in dilute solution," J. Chem. Phys. 104, 6355 (1996). 
${ }^{47}$ P. Ahlrichs and B. Dünweg, "Simulation of a single polymer chain in solution by combining lattice Boltzmann and molecular dynamics," J. Chem. Phys. 111, 8225 (1999).

${ }^{48}$ A. Z. Akcasu, M. Benmouna, and C. C. Han, "Interpretation of dynamic scattering from polymer solutions," Polymer 21, 866-890 (1980).
${ }^{49}$ H. Kobayashi and R. G. Winkler, "Universal conformational properties of polymers in ionic nanogels," Sci. Rep. 6, 19836 (2016).

${ }^{50}$ S. Maccarrone, O. Mergel, F. A. Plamper, O. Holderer, and D. Richter, "Electrostatic effects on the internal dynamics of redox-sensitive microgel systems," Macromolecules 49, 1911-1917 (2016). 